\title{
Hypertension in anaesthesia
}

\author{
CONTENTS \\ Aetiology \\ Pathophysiology \\ Treatment/implications for the anaesthetist \\ Diuretics \\ Adrenergic beta-receptor antagonists \\ Alpha-2 adrenergic receptor agonists \\ Peripheral vasolidators \\ Adrenergic neurone blockers \\ Drugs affecting angiotensir. II \\ Calcium entry blocking drugs \\ Anaesthetic management \\ Preoperative assessment \\ Perioperative monitoring \\ Premedication \\ Induction and maintenance \\ Intraoperative hypertension \\ Postoperative hypertension
}

Arterial hypertension affects $15-20$ per cent of the North American population. Hypertension is defined as a systolic blood pressure greater than $160 \mathrm{mmHg}$ or a diastolic blood pressure greater than $95 \mathrm{mmHg}$. There is also evidence that patients with "borderline hypertension" that is, a blood pressure between $140 / 90$ and 160/95 may have an increased risk of developing the complications of hypertension.

\section{Aetiology}

The aetiology of hypertension is classified as either Primary or Secondary and is outlined in Table I. ${ }^{1}$

Primary or essential hypertension presents independently of any known specific pathology and accounts for 90 per cent of all cases of hypertension.

Factors which may contributc to the development of essential hypertension include heredity, obesity, salt intake, alcohol consumption and psychological factors such as stress.

\section{Pathophysiology of hypertension}

The following are the main pathophysiological changes associated with hypertension. ${ }^{2}$
1. Hypertension is due to an increased systemic vascular resistance. Cardiac output is normal. The high resistance is due to increased thiskening of the arterial wall resulting in decreased intraluminal diameter. The process is termed arteriosclerosis.

2. Although the hypertensive patient's arterioles are capable of vasoconstriction and vasodilatation, the degree of change is greater than in the normotensive patient. Therefore, the pressure response to stimuli such as endotracheal intubation may be much greater than normal.

3. Atheromatous vascular disease is common in hypertensive patients. Hypertension does not cause atheroma but it does accelerate atheromatous degeneration.

4. Effects on the heart: Left ventricular hypertrophy may result due to the increased work which the ventricle has to perform to overcome the increased systemic vascular resistance. This may lead to left ventricular failure.

Coronary artery disease is common in hypertensive patients. Approximately $30-60$ per cent of hypertensive patients die of myocardial infarction. Both hypotension and acceleration of the hypertensive process may result in myocardial infarction.

5. Effects on the brain: The autoregulary pressure limits of cerebral blood flow are shilted upwards in the hypertensive patient.

Hypotension may result in cerebral infarction.

Sustained hypertension or acceleration of the hypertensive process may result in cerebral haemorrhage.

6. Effects on the kidney: Hypertension results in thickening of the renal arterioles resulting in renal nephrosclerosis. Renal insufficiency may result. As in the brain, the autoregulatory pressure limits of renal blood flow are shifted upwards in the hyper-

From the Department of Anacsthesia, Faculty of Medicine, Memorial University of Newfoundland.

Address correspondence to: $\mathrm{Dr}$. D. Reid,

Department of Anaesthesia, The Health Sciences Centre, St. John's, Newfoundland, A1B 3V6. 
tensive patient. Hypotension may result in worsening renal insufficiency going on to renal failure.

7. Effects on the retina: Fundoscopic examination in the hypertensive patient may revel haemorrhages and exudates. Retinal detachment is also a complication of longstanding hypertension.

8. Effects on the aorta: Dissection of the aorta may occur with longstanding hypertension.

\section{Treatment of hypertension}

The major groups of drugs used in the treatment of hypertension will be reviewed. Ganglion blocking agents are now uncommonly used in the treatment of hypertension and therefore have been omitted. Alpha adrenergic receptor blockers such as phenoxybenzamine and phentolamine are usually reserved for the management of patients with phaeochromocytoma and have also been omitted from this review.

1. Diuretics (e.g., chlorthalidone, hydrochlorothiazide, spironolactone)

These are particularly effective in patients with low renin activity. They are sometimes used alone or in combination with other agents. The mechanism by which diuretics lower the blood pressure is unclear. Mechanisms suggested include an initial reduction in blood volume and a decreased sensitivity of arterioles to catecholamines.

\section{Implications for the anaesthetist}

Diuretic therapy results in a five to ten per cent reduction in blood volume. ${ }^{2}$ Although this returns to normal after prolonged administration, there is the possibility of hypotension occurring during anaesthesia.

There is depletion of total body potassium associated with the administration of many diuretics. The plasma level may or may not be within normal limits. Potential interactions of hypokalaemia with anaesthesia include potentiation of non-depolarising muscle relaxants, ventricular dysrhythmias and potentiation of digitalis toxicity,

Although plasma potassium levels do not reliably indicate total body potassium it is the only measurement available to the practicing physician. Our guidelines are a minimum serum potassium of $3.5 \mathrm{mEq} / \mathrm{L}$ in a digitalised patient and $3.0 \mathrm{mEq} / \mathrm{L}$ in all other patients.

Hyperkalaemia is a possibility in patients taking "potassium sparing" diuretics, e.g., spironolac-
TABLE I Classification of arterial hypertension ${ }^{1}$

I. Systolic hypertension with wide pulse pressure

A. Decreased compliance of aorta (arteriosclerosis)

B. Increased stroke volume

1. Anteriovenous fistula

2. Thyrotoxicosis

3. Hyperkinetic heart disease

4. Fever

5. Psychogenic factors

6. Aortic regurgitation

7. Patent ductus arteriosus

II. Systolic and diastolic hyperension (increased peripheral vascular resistance)

A. Renal

1. Chronic pyelonephritis

2. Acute and chronic glomerulonephritis

3. Polycystic renal disease

4. Renovascular stenosis or renal infarction

5. Most other severe renal disease (arteriolar nephrosclerosis, diabetic neophropathy, etc.)

6. Renin-producing tumars

B. Endocrine

1. Oral contraceptives

2. Adrenocortical hyperfunction

a. Cushing's disease and syndrome

b. Primary hyperaldosteronism

c. Congenital or hereditary adrenogenital syndromes

(17 $\alpha$-hydrosylase and IL $\beta$-hydroxylase defects)

3. Phaeochromocytoma

4. Myxoedcms

5. Acromegaly

C. Neurogenic

1. Psychogenic

2. "Diencephalic syndrome"

3. Familial dysautonomia (Riley-Day)

4. Poliomyelitis (bulbar)

5. Polyneuritis (acute porphyria, lead poisoning)

6. Increased intracranial pressuse (acute)

7. Spinal cord section

D. Miscellaneous

1. Coarctation of aorta

2. Increased intravascular volume (excessive transfusion, polycythemia vera)

3. Polyarteritis nodosa

4. Hypercalcemia

E. Unknown aetiology

1. Essential hypertension ( $>90 \%$ of all cases of hypertersion)

2. Toxemia of pregnancy

3. Acute intermittent porphyria

tone. Hyperkalaemia may be worsened during anaesthesia by the administration of succinylcholine. Under these conditions the ensuing hyperkalaemia may result in ventricular dysrhythmias. We suggest an upper limit of $5.5 \mathrm{mEq} / \mathrm{L}$ as being acceptable in the elective surgical patient. 
Hyperglycaemia and hyperurcaemia can both occur during diusetic therapy.

\section{Adrenergic beta-receptor antagonists}

These are more commonly referred to as "beta blockers."

These agents reduce the force of myocardial contraction and hence reduce cardiac output. This is probably the mechanism by which they reduce blood pressure although they also possess anti-renin activity.

The fact that beta-blockers reduce cardiac output contraindicates their use in patients with cardiac failure.

\section{Principle of cardioselectivity}

Beta receptors are classified into two distinct types, beta 1 and beta $2 .^{3}$ Beta 1 receptors are responsible for cardioacceleration, increased myocardial contractility and lipolysis. Beta 2 receptors are responsible for arteriolar vasodilatation, bronchodilatation, intestinal, uterine and bladder relaxation, glycogenolysis and calorogenesis. Increased renin secretion is a function of both beta 1 and beta 2 receptors. The classification of beta receptors had led to the development of antagonist drugs which are relatively selective at beta 1 or beta 2 receptors. ${ }^{4}$

The beta blockers are therefore classified as either selective or non-selective. In low dosage, beta 1 selective blockers block beta 1 receptors only. This is also termed cardioselectivity. Nonselective beta blockers block beta 1 and beta 2 receptors. In high dosage selective beta 1 blockers will also block both beta 1 and beta 2 receptors.

There are several advantages to the use of beta 1 selective blockers. They are potentially safer in patients with chronic obstructive lung disease since the beta 2 receptors are still available to mediate bronchodilatation. Peripheral vasodilatation is still possible which may be of advantage in the treatment of hypertension.

In diabetic patients the beta 2 receptor can still mediate epinephrine induced glycogenolysis in response to hypoglycaemia even though the cardiovascular signs of hyperglycaemia may be abolished by the beta 1 blockade.

A list of available beta receptor blockers is given in Table II.

It can be seen from Table II that apart from atenolol and nadolol most beta blockers have short plasma half-lives. However, it must be pointed out that there is a lack of correlation between plasma half-life and pharmacological effect, which is longer than the plasma half-life might suggest.

The mode of elimination of beta blockers may determine the selection of agent in patients with hepatic or renal disease.

\section{Implications for the anaesthetist}

Beta blockers should not be discontinued prior to surgery but should be administered up to and including the day of surgery and re-instituted postoperatively as soon as possible.

There is evidence that abrupt withdrawal of these agents may result in some patients cleveloping an increased sensitivity to endogenous epinephrine and norepinephrine resulting in hypertension, tachycardia, and myocardial ischaemia. ${ }^{5}$ During the perioperative period various events such as anxiety, the surgical incision and tracheal intubation will result in increased release of catecholamines. In the partially "beta blocked" patient or in the patient whose drugs have been stopped prematurely in the preoperative period, it may be necessary to administer beta blockers to treat the ensuring hypertension, tachycardia and myocardial ischaemia. Propranolol $1-3 \mathrm{mg}$. may be given at the rate of $1 \mathrm{mg} / \mathrm{min}$ to gain control of the situation.

It is important for the anaesthetist 10 "tailor" his technique to minimize catecholamine release secondary to noxjous stimuli such as endotracheal intubation.

There is no doubt that the effects of anaesthesia and beta blockers are additive. The evidence in the current literature ${ }^{6}$ suggests that:

(a) isoflurane, narcotics and halothane in that order are the agents of choice in the beta blocked patient;

(b) the status of enflurane is undecided;

(c) trichlorethylene, cyclopropane, ether and methoxyfluorane should not be used unless there is an absolute indication.

Hypotension and bradycardia may result due to the additive effects of beta blockers and anesthetic agents and it may be necessary to reverse the beta blockade.

Initially atropine $0.4 \mathrm{mg}$ repeated every five minutes to a maximum dose of $2.0 \mathrm{mg}$ may be given. If this fails to resolve the problem, calcium chloride $5 \mathrm{ml}$ of ten per cent solution repeated every ten minutes may be given. If atropine and calcium chloride fail to resolve the problem, cardiac pacing 
TABLE Il Pharmacological features of beta receptor blockers

\begin{tabular}{|c|c|c|c|c|c|c|c|}
\hline Feature & Propranolol & Metoprolul & Nadolol & Atenolol & Pindolol & Timolol & Oxprenolol \\
\hline Beta, selectivity & 0 & + & 0 & + & 0 & 0 & 0 \\
\hline \multicolumn{8}{|l|}{ Beta $_{1}$ blocking potency } \\
\hline (propranol 1) & 1.0 & 1.0 & 1.0 & 1.0 & 6.0 & 6.0 & 2.0 \\
\hline Half life (hours) (oral dose) & $3.5-6$ & $3-4$ & $14-24$ & $6-9$ & $3-4$ & $3-4$ & $1.3-1.5$ \\
\hline Elimination & Liver & Liver & Kidney & Kidney & Kidney and liver & Liver & Kidney \\
\hline Active metabolites & Yes & No & No & No & No & No & No \\
\hline
\end{tabular}

or an isoproterenal infusion given at a rate of $2-20 \mu \mathrm{g} / \mathrm{min}$ depending on response, may be necessary.

\section{Alpha $\mathrm{a}_{2}$ adrenergic receptor agonists}

Two types of alpha adrenergic receptors have been described. They are classified as alpha $a_{1}$ and alpha receptors.

Alpha, receptors, previously called alpha receptors, are postsynaptic in relation to the adrenergic nerve terminal and mediate arterial vasoconstriction.

Alpha $_{2}$ receptors are the predominant alpha receptor in the brain, and are also found on the presynaptic portion of the adrenergic nerve terminal. Centrally alpha ${ }_{2}$ receptor stimulation depresses sympathetic activity and enhances vagal tone, while peripherally alph $\mathrm{a}_{2}$ stimulation inhibits norepinephrine release from the adrenergic nerve terminal.

Alpha-methyldopa has both central and peripheral actions. It is converted to alpha-methyl norepinephrine which in turn stimulates alpha $a_{2}$ receptors. Peripherally alpha-methyl norepinephrine is stored in adrenergic nerve endings. This is released in response to nerve stimulation, stimulating peripheral alpha $_{2}$ receptors. Centrally alpha-methyl norepinephrine acts on alpha ${ }_{2}$ receptors in the vasomotor centre in the medulla.

The overall effect is decreased sympathetic activity, increased vagal activity and lower plasma norepinephrine and epinephrine levels. Renin secretion is also decreased. Alpha-methyldopa maintains or increases renal blood flow and is therefore a good choice in patients with renal insufficiency.

Clonidine is an alpha 2 receptor agonist and like alpha-methyl norepinephrine acts on the alpha receptors in the vasomotor centre in the medulla.

The peripheral effect is minimal. The overall effect is decreased sympathetic activity, increased vagal tone and decreased plasma levels of epinephrine and norepinephrine. Renin secretion from the kidney is also decreased.

\section{Implications for the angesthetist}

These drugs should not be stopped prior to surgery. Patients receiving alpha-methyldopa of clonidine may have a sinus bradycardia due to parasympathetic predominance. A decrease in MAC (minimum alveolar concentration) of about 16 per cent has been noted in experimental animals. ${ }^{7}$

A positive Coombs test occurs in about 20 per cent of patients treated with alpha-methyldopa. This may pose problems in cross-matching blood for surgery.

Dementia is a noted unwanted effect of alphamethyldopa treatment. This is due to dopamine depletion in the central nervous system. This effect may be potentiated by butyrophenones such as droperidol which should be avoided in such patients

It is important that clonidine not be stopped abruptly since severe rebound hypertension may occur. ${ }^{8}$ This withdrawal syndrome is most severe in patients on high dosage of clonidine, that is, more than $1.2 \mathrm{mg}$ daily. The withdrawal syndrome begins about 18 hours after the drug has been stopped. The patients develop sweating, apprehension headache, flushing and tremulousness. In such circumstances intravenous diazoxide or hydralazine plus a beta blocker may be necessary to control the hydralazine syndrome.

\section{Peripheral vasodilators}

These agents act directly on vascular smooth muscle.

Hydralazine - has a short plasma half-life of 1.7-3 hours but it may bind to vascular smooth 
muscle and exert its effect for 1-6 days. Hydralazine also causes an increase in renal blood flow and therefore is a useful agent in the treatment of those patients who have co-existing renal insufficiency.

Since baroreceptor responses remain intact with hydralazine, increased sympathetic discharge will result in tachycardia and increased cardiac work. This may be detrimental to the patient with coronary artery insufficiency and may also offset the hypotensive effect. For this reason a beta receptor blocker is usually prescribed at the same time.

Hydralazine is also available in parenteral form and may be used for the treatment of acute hypertension in a dosage of $10-40 \mathrm{mg}$ IV by slow injection. There may be no response for ten minutes.

Prazosin - As well as having a direct action on vascular smooth muscle, it is also an alpha adrenergic receptor blocker. It has a plasma halflife of 2-3 hours but the hypotensive effect lasts for six hours. Like hydralazine it is often used in combination with a beta receptor blocker to abolish reflex tachycardia. This agent is usually reserved for patients who cannot tolerate hydralazine. It has been notcd that about one per cent of patients taking prazosin develop profound hypotension within 30 90 minutes of the first dose. The first dosage is therefore usually limited to $0.5 \mathrm{mg}$ given at bedtime.

Minoxidil - has a plasma half-life of 4.2 hours but the hypotensive effect is present for three days. Treatment results in a reflex tachycardia as described with hydralazine and prazosin and therefore a beta receptor blocker may need to be prescribed at the same time.

Fluid retention is also a problem with minoxidil, necessitating the concurrent administration of a potent diuretic such as furosemide.

Diazoxide - is used for the rapid control of acute severe hypertension. It is given intravenously in doses up to $300 \mathrm{mg}$ over 30 seconds. A response usually occurs within five minutes. A second dose may be given if the first fails to produce a response after 30 minutes.

The common unwanted effects of this agent are fuid retention, which may require treatment with a diuretic, and hyperglycaemia. Blood glucose levels should be measured in patients receiving this agent.

Implications for the anaesthetist

Hydralazine, prazosin and minoxidil should be continued up to and including the day of surgery and should be re-instituted as soon as possible postoperatively. The anaesthetist should be aware that hypotension may occur in combination with vasodilating anaesthetic agents.

\section{Adrenergic neurone blockers}

Reserpine prevents norepinephrine accumulation in the storage vesicles of the adrenergic neurones. Norepinephrine then collects in the nerve ending where it is oxidised by monoamine oxidase.

Reserpine also acts centrally where it depletes stores of both catecholamines and 5-hydroxytryptamine. This results in many of the unwanted effects of reserpine such as mental depression, sedation and Parkinsonian-type rigidity. Reserpine is not usually used alone in the treatment of hypertension but as part of combined therapy.

Guanethidine is taken into the sympathetic nerve ending in the same way as norepinephrine. It enters the storage vesicles causing norepinephrine release. Norepinephrine stores are therefore depleted.

The decreased reponsiveness of the resistance and capacitance vessels resulting in decreased venous return, decreased cardiac output and peripheral vasodilatation are most marked when the patient is in the upright position. This results in the patient developing postural hypotension.

Debrisoquine is structurally related to guanethidine. Its main action is the prevention of release of norepinephrine from storage granules in the adrenergic neurone. Debrisoquine is also a monoamine oxidase inhibitor but this action is unlikely to be clinically significant.

\section{Implications for the anaesthetist}

Reserpine reduces MAC by about 14 per cent, ${ }^{7}$ whereas guanethidine does not cross the blood brain barrier and does not cause the central effects seen with reserpine.

Drugs which prevent uptake of norepinephrine will also prevent uptake of guanethidine. These include ketamine, cocaine, pancuronium and tricyclic antidepressants. ${ }^{6}$ This may result in hypertension.

Patients receiving these drugs have an altered response to sympathomimetic drugs. On the one hand direct acting sympathomimetics such as methoxamine may produce an exaggerated response due to alpha receptor hypersensitivity while indirect 
acting sympathomimetics such as ephedrine may have a decreased effect. Should hypotension occur during anaesthesia in such patients a small dose of a direct acting sympathomimetic such as methoxamine $0.25 \mathrm{mg}$ repeated as necessary may be effective in restoring blood pressure to reasonable levels.

\section{Drugs affecting angiotesin II}

Captopril inhibits converting enzyme which is involved in the conversion of angiotensin I to angiotensin II. This results in a fall in peripheral arterial resistance with increase in renal blood flow without change in cardiac output. Aldosterone secretion is also decreased, promoting salt and water loss.

There are no communications in the literature to date to indicate any specific implications for the anaesthetist regarding the use of captopril.

\section{Calcium entry blocking drugs ${ }^{9}$}

All excitable cell membranes contain both fast and slow channels. The former allows the influx of sodium and the latter the influx of calcium during depolarisation. Both of these channels have inner and outer gates.

The movement of calcium into the cell in skeletal, cardiac and smooth muscle is essential for muscle contraction. Calcium entry is also essential for depolarisation in the automatic cells of the heart such as the sino-atrial and atrio-ventricular nodes.

Blocking of calcium channels in skeletal muscle does not adversely affect contraction since there are large intracellular stores available for use. However, cardiac and smooth muscle and the automatic cells of the heart require the influx of calcium to function normally.

Verapamil blocks the entry of calcium into the cell at the inner gate of the slow channel and nifedipine blocks the entry of calcium into the cell at the outer gate of the slow channel.

Nifedipine is an effective antihypertensive. In addition to its vasodilating effect it also decreases plasma renin activity.

\section{Implications for the anaesthetist}

These drugs should not be stopped prior to surgery. However, the potential interaction with anaesthetic agents warrants caution on the part of the anaesthetist.

Administration of these agents intraoperatively may result in cardiovascular collapse due to the interaction with anaesthetic agents, which in themselves cause myocardial depression and peripheral vasodilation. This may be treated with calcium chloride or isoproterenol. Heart block may occur specifically with verapamil.

\section{Anaesthetic management}

\section{Pre-operative assessment}

Part of the assessment of patient risk and essential components of anaesthetic management are the review of primarily those organ systems damaged by longstanding hypertension; namely the brain, heart and kidneys, by history, physical examination and clinical investigations.

The physical signs of a cerebrovascular accident may be visible with or without a carotid bruit, but transient cerebral ischaemia can only be diagnosed by history. An elevated systolic blood pressure is the most frequent precursor of cerebral infarction, arteriosclerosis and renal vascular disease.

Proteinuria, abnormal urinary sediment and inability to concentrate the urine denotes renal insufficiency. Blood urea nitrogen and creatinine levels will be elevated at a point where less than $30 \mathrm{per}$ cent residual function remains. The EKG and CXR are most important in assessing heart rhythm, state of the myocardial wall, and presence of congestive heart failure, and compliment close interrogation and physical examination. ${ }^{10}$

A history of congestive failure is a major predictor of cardiac problems. These patients are in a critical fluid balance and have a very narrow safety limit. The physical finding of marked orthostatic hypotension denoting dehydration should be sought. A diastolic pressure greater than $100 \mathrm{mmHg}$ is the hallmark of renal, endocrine and essential hypertension and this group of patients is more likely to benefit from preoperative blood pressure control. As a rule, anaesthetic agents are myocardial depressants and lower peripheral vascular resistance placing these patients at risk of hypotension especially on induction of anaesthesia.

Earlier studies by Prys-Roberts concluded that the reflex pressor response on intubation has the same incidence in both treated and non-treated groups of hypertensives and may be limited but not prevented by prior medical therapy. ${ }^{11}$ In a recent update, Goldman and Caldera recommended that the optimal preoperative care of the hypertensive is tight blood pressure control but they state that the 
incidental finding of a systolic pressure less than $180 \mathrm{mmHg}$ and diastolic pressure no greater than $110 \mathrm{mmHg}$ is no longer a contraindication to surgery and that close patient monitoring for hyperand hypotension perioperatively renders patient risk equal to treated, but poorly controlled hypertensives. ${ }^{12}$ The severe hypertension must be controlled before anaesthesia. ${ }^{13} \mathrm{~A}$ triad of physical findings denotes this readily identifiable high risk subgroup. 1. A diastolic pressure greater than $120 \mathrm{mmHg}$.

2. Grade III or IV retinopathy with haemorrhage exudates and papilloedema.

3. An elevated BUN or creatinine.

\section{Perioperative Monitoring}

Central venous, arterial and pulmonary artery pressure monitoring are reserved for the patient with poor myocardial function and past history of congestive heart failure, or in the emergency situation faced with the presence of heart failure or uncontrolled severe hypertension requiring immediate blood pressure control with potent vasodilators, and necessitating manipulation of the intravascular volume by estimating pulmonary capillary wedge pressure. In the moderately hypertensive patient the minimal acceptable monitoring includes continuous electrocardiographic display preferably lead $V_{5}$, and intermittent blood pressure measurement by sphygmomanometer. ${ }^{16}$

\section{Premedication}

Preoperative anxiety, anaesthetic induction and intubation, skin incision, surgical manipulation, anaesthetic reversal and extubation represent periods of high sympathetic activity during which elevated plasma catecholamine levels correspond to mean arterial pressure resulting in cumulative stress in the cardiovascular system. Good preoperative sedation is provided by morphine $0.1 \mathrm{mg} \cdot \mathrm{kg}^{-1}$ in combination with a phenothiazine. Benzodiazepines do not alter cardiovascular stability and lorazepam particularly produces retrograde amnesia in higher clinical dosage. Glycopyrrolate has no central excitatory effect as the polarised molecule does not cross the blood brain barrier. It has superseded the other cholinergics, atropine and hyoscine, as it is more effective in preventing gastric acid secretion with minimal tachycardia and discomfort of a dry mouth.

\section{Anaesthetic induction and maintenance}

Using a non-invasive method with radioisotope red cell labelling left ventricular performance was shown to be transiently depressed in all patients healthy and otherwise during intubation, but remained so in about 25 per cent of patients with myocardial disease. Varjous induction regimens attempt to attenuate this sympathetic pressure reflex with deep inhalational anaesthesia, fentanyl $30 \mu \mathrm{g}$. $\mathrm{kg}^{-1}$ as induction agent, or supplementing an induction agent with xylocaine, both topical or intravenous, or small boluses of nitroprusside or nitroglycerine $(100-200 \mu \mathrm{g}) .^{14}$

Anaesthetic maintenance can be achieved with little myocardial depression using intermittent boluses or continuous infusion of narcotic agent and nitrous oxide and muscle relaxant, utilizing crystalloid to maintain pressure rather than vasopressors and inhalational agents to control high levels of systemic pressure. ${ }^{10}$ Choice of narcotic agent is arbitrary. Fentanyl produces minimal myocardial depression and lacks the peripheral vascular effects associated with histamine release. The observed drop in heart rate is beneficial to the ischaemic myocardium.

The dose-related myocardial depression and decreased $A \cdot V$ conduction of halothane may benefit the patient with ischaemic heart disease. Enflurane produces most myocardial depression. Isoflurane produces least depression and maintains cardiac output by potent peripheral vasodilalion, heart rate rises possibly by a direct beta stimulant effect. Isoflurance also minimally sensitises the heart to catecholamines. Nitrous oxide has a direct myocardial depressant effect and raises peripheral resistance. Nitrous oxide and diazepam potentiate the myocardial depression of narcotic analgesics. The combination is not recommended for patients with compromised myocardial function.

Droperidol, a butyrophenone derivative, has alpha adrenergic blocking properties and enhances the central effects of narcotics. Its antiemetic and sedative actions are prolonged into the postoperative period.

Muscle relaxants alter cardiovascular stability by autonomic stimulation and depression, histamine release and changing serum $\mathrm{K}^{+}$concentration.

Until the promised release of new agents, metacurine, a derivative of curare, lacking its histamine releasing and ganglionic blockage properties in low 
dose, and pancuronium whose tachycardia response offsets a narcotic induced bradycardia, are the best agents available. Use of succinylcholine is associated with a high incidence of arrhythmias especially in the elderly and should be reserved for rapid or difficult intubations.

\section{Treatment of intraoperative hypertension}

Both hypertension and its treatment can produce ischaemic myocardial injury. Systemic pressure will decrease in response to manipulation of myocardial contractility and the lowering of preload, afterload and heart rate, the latter being the most important determinant of myocardial $\mathrm{O}_{2}$ consumption. Coronary perfusion pressure and myocardial blood flow drop in response to a lowering of diastolic pressure. ${ }^{17}$

Hydralazine, a potent peripheral vasodilator, ,will decrease systemic pressure within 10-15 minutes of a dose of $5-10 \mathrm{mg}$ intravenously and $30-60$ minutes of $10-20 \mathrm{mg}$ given intramuscularly. A reflex tachycardia is common and is controllable with propranolol and is useful if systemic pressure rises despite increasing concentrations of inhalational agent and intravenous propranolol. Alpha receptor antagonists are reserved for the treatment of phaeochromocytoma. ${ }^{\text {is }}$

Sodium nitroprusside is nearly always successful in decreasing arterial pressure. After injection it is converted to cyanide and then to thiocyanate in the liver. Thiocyanate is excreted in the urine and accumulates in patients with renal failure. The dosage of sodium nitroprusside is $0.5-10 \mu \mathrm{g} \cdot \mathrm{kg}^{-1}$. $\mathrm{min}^{-1}$ and if there is no response at a higher dosage level, the agent should be discontinued. It preferentially dilates the arterial side of the circulation and may result in decreased coronary perfusion.

Nitrates are less polent vasodilators. Nitroglycerine promotes pooling in the venous system. The preload is reduced, decreasing ventricular volume, wall distension and therefore myocardial $\mathrm{O}_{2}$ demand. Myocardial blood flow may be secondarily enhanced with greater flow to poorly perfused areas and this agent is preferrable to nitroprusside for use in patients with ischaemic heart disease. If the preload is maintained by volume infusion, an afterload effect will predominate.

Major cardiovascular surgery, renal endocrine and neurological disease increase the incidence of postoperative hypertension.

\section{Postoperative hypertension}

Uncontrolled hypertensive patients have exaggerated responses to the high sympathetic activity brought on by hypoxia, hypercarbia and pain. Continuation of preoperative antihypertensive therapy decreases the incidence of postoperative hypertension to that of the normotensive patient. In the postoperative period anticipation of a higher incidence of hypertensive episodes and their sequalae, congestive failure, myocardial ischaemia, arrhythmias, correction of transient renal insufficiency by fluid therapy, adequate postoperative analgesia and switching to long-acting oral antihypertensives will minimise the risk of cardiovascularcomplications. ${ }^{18}$

\section{References}

1 Harrison's Principles of Internal Medicine. 10th ed. McGraw-Hill Book Co., 1983, New York.

2 Prys-Roberts $C$. Hypertension, ischaemic heart disease and anaesthesia. International Anaesthesiology Clinics. 18: 4, Winter 1980

3 Guyton AC. Textbook of Medical Physiology. 6th ed. 1981. W.B. Saunders Co.

4 Frishman $W H$. $\beta$-adrenoceptor antagonists. New Drugs and New Indications. N Engl J Med 1981; 305: 500-06.

5 Boudoulas $\boldsymbol{H}$ et al. Hypersensitivity to Adrenergic stimulation after propranolol withdrawal in normal subjects. Ann Intern Med 1977; 87: 433.

6 Ty Smith N, Miller RD, Corbascio AN. Drug Interactions in Anesthesia. Lea \& Febiger, Philadclphia, 1981.

7 Miller RD. Way WL, Eger, EIII The effect of alpha-methyldopa, reserpine, guanethidine, and iproniazid on minimal alveolar anesthetic requirement (M.A.C.). Anesthesiology 1969; 29: 1153.

8 Brodsky JB, Bravo, J J. Acute post-operative clonidine withdrawal syndrome. Anesthesiology 1976; 44: 519 .

9 Merin RG. Calcium "Slow" Channel Blocking Drugs. A.S.A. Refresher Courses vol. 10, ch. 12, 1982.

10 Vertes $V$, Goldberg $G$. The pre-operative patient with hypertension. Med. Clin. N. Am. 1070; 63: 1299.

11 Prys-Roberts C. Hypertension and anaesthesia fifty years on (editorial). Anesthesialogy 1979; 50: 281.

12 Goldman $L$, Coldera DL. Risks of general anaesthe- 
sia and elective operation in the hypentensive patient. Anesthesiology 1979; 50: 285.

13 Prys-Roberts $C$. Vascular disease and anaesthesia (editorial). Br. J Anaesth 1981; 53: 7.

14 Nitroprusside and other short acting hypotensive agents. Int. Anaesth. Clin. 1978; 16: 2.

15 Marshall AJ. Drug therapy of hypertension and ischaemic heart disease. Br. J. Anaesth 1981; 53 : 697.

16 Prys-Roberts $C$. Cardiovascular monitoring in patients with vascular disease. Br. J Anaesth 1981; 53: 767.

17 Miller ED. Hypcrtension and its significance to anaesthesia. ASA Refresher Course Lectures 1982. p. 202.

18 Prys-Roberts $C$. Anaesthetic considerations in the hypertensive patient. ASA Refreser Course Lcctures 1979, p. 139. 